\title{
HISTOPATOLOGÍA DEL ENDOMETRIO EN SANGRADO UTERINO EN LA POSTMENOPAUSIA- INSTITUTO NACIONAL MATERNO PERINATAL
}

\author{
Félix Dasio Ayala Peralta ${ }^{1}$, César Carranza Asmat² ${ }^{2}$ David Félix Calle Zurita ${ }^{3}$.
}

\begin{abstract}
RESUMEN
Objetivo. Determinar la prevalencia del tipo histológico del endometrio en pacientes con sangrado uterino en la postmenopausia. Materiales y métodos. Estudio observacional, retrospectivo, transversal. Se incluyeron 40 casos que tuvieron estudio histopatológico de pacientes que presentaron sangrado uterino cuyas edades comprendidas fueron de 50 a más años de edad. Se revisaron los hallazgos histopatológicos. Resultados. La mayoría de casos corresponden a la edad de 50 a 59 años $(77 \%)$. El tipo histológico más frecuente corresponde a endometrio normal en $45 \%$ (18 casos), seguida de $15 \%$ ( 6 casos) cáncer endometrial, $13 \%$ ( 5 casos) endometrio atrófico, $7 \%$ ( 3 casos) pólipo endometrial y $5 \%$ ( 2 casos) hiperplasia endometrial. La atrofia endometrial se halló en todos los grupos etarios estudiados, mientras la hiperplasia endometrial y el pólipo endometrial se presentan con más frecuencia entre 50 a 59 años de edad. La histopatología del cáncer endometrial fue carcinoma endometrial ( 2 casos), carcinoma epidermoide ( 2 casos), adenocarcinoma ( 1 caso) y un caso de carcinoma seroso papilar. Conclusión. La histopatología del endometrio en sangrado uterino postmenopáusico corresponden en la mayoría a endometrio normal seguida de cáncer endometrial, endometrio atrófico y pólipo endometrial.
\end{abstract}

Palabras clave: Sangrado postmenopáusico; Histopatología; Endometrio ( Fuente: DeCs BIREME).

\section{HISTOPATHOLOGY OF ENDOMETRIAL UTERINE BLEEDING POSTMENOPAUSAL- NATIONAL MATERNAL PERINATAL INSTITUTE}

\begin{abstract}
Objective. Determine the prevalence of endometrial histology in patients with postmenopausal uterine bleeding. Materials and methods. Observational, retrospective, cross. Study 40 cases had histopathological study of patients who had uterine bleeding whose ages were 50 or more years of age were included. Pathological findings were reviewed. Results. Most cases correspond to the age of $50-59$ years $(77 \%)$. The most common histological type corresponds to normal endometrium in $45 \%$ ( 18 cases), followed by $15 \%$ ( 6 cases) endometrial cancer, $13 \%$ (5 cases) atrophic endometrium, $7 \%$ ( 3 cases) endometrial polyp and $5 \%$ ( 2 cases) endometrial hyperplasia. Endometrial atrophy was found in all age groups studied, while the endometrial hyperplasia and endometrial polyps occur most frequently between 50-59 years old. The histopathology of endometrial cancer was endometrial carcinoma ( 2 cases), squamous cell carcinoma ( 2 cases), adenocarcinoma ( 1 case) and a case of papillary serous carcinoma. Conclusion. Histopathology of the uterine endometrium in postmenopausal bleeding correspond in most normal endometrium followed by endometrial cancer, atrophic endometrium and endometrial polyp.
\end{abstract}

Key words: Postmenopausal bleeding; Histopathology; Endometrium ( Source: MeSH NLM).

\section{INTRODUCCIÓN}

El sangrado uterino en la posmenopausia se refiere a cualquier sangrado uterino que se presenta después de un año de amenorrea en una mujer en edad postmenopáusica ${ }^{1,2}$.

Dicho sangrado vaginal se produce en aproximadamente 4 a 11 por ciento de las mujeres posmenopáusicas ${ }^{3-6}$.

Todas las mujeres posmenopáusicas con sangrado uterino inesperado deben ser evaluados para descartar cáncer endometrial ya que esta enfermedad es la causa de la hemorragia en aproximadamente el 10 por ciento ${ }^{1,7-10}$,. Sin embargo, la causa más común de sangrado postmenopáusico es la atrofia vaginal o endometrial ${ }^{11,12}$. Asimismo, cabe señalar que en los primeros años de la postmenopausia son también causas comunes, las hiperplasias endometriales, pólipos y miomas submucosos ${ }^{12-14}$.

La evaluación endometrial mediante la biopsia endometrial o ultrasonido transvaginal es fundamental y es el componente clave para el diagnóstico de lesión premaligna endometrial o cáncer de endometrio en mujeres con sangrado uterino anormal que se pueden utilizar como una prueba inicial y prácticamente es el estándar de oro para evaluar el endometrio en sangrado postmenopáusico.

La ecografía transvaginal permite la medición del grosor endometrial. En las pacientes con endometrio engrosado amerita la valoración mediante biopsia endometrial con

Médico GínecoObstetra. Magíster en Salud Reproductiva. Profesor de la Facultad de Medicina Humana de la Universidad San Martín de Porres y de Ciencias de la Salud de la Universidad Norbert Wiener. Director de la Oficina de Cooperación Científica Internacional-Instituto Nacional Materno Perinatal de Lima-Perú.

2 Médico GínecoObstetra. Profesor Asociado de la Facultad de Medicina de la Universidad Nacional Mayor de San Marcos. Director Ejecutivo de la Oficina Ejecutiva de Apoyo a la Investigación y Docencia Especializada-Instituto Nacional Materno Perinatal de Lima-Perú.

3 Médico Anatomo-Patólogo. Profesor de la Facultad de Medicina Humana de la Universidad Federico Villarreal. Jefe del Departamento de PatologíaInstituto Nacional Materno Perinatal de Lima-Perú. 
aspiración manual endouterina (AMEU), legrado uterino instrumental o histeroscopía. Se considera el estudio con endometrio mayor a $14 \mathrm{~mm}$ en mujeres premenopáusicas, mayor a $5 \mathrm{~mm}$ en mujeres menopáusicas sin tratamiento de reemplazo hormonal, y mayor a $8 \mathrm{~mm}$ en mujeres postmenopáusicas que se encuentran con terapia hormonal ${ }^{15,16}$.

En relación al tipo de histología endometrial, según la OMS, la hiperplasia endometrial se clasifica en hiperplasia simple, compleja (según la ausencia/presencia respectivamente, de anomalías arquitectónicas), atípica (presencia de atipia celular-nuclear), atípica simple y atípica compleja. La neoplasia intraepitelial endometrial hace referencia a dos categorías de hiperplasia: endometrio policlonal normal con respuesta difusa a un ambiente hormonal anormal, y lesiones monoclonales con proliferación intrínseca focal y que significan un alto riesgo de adenocarcinoma ${ }^{15,17}$.

Asimismo, más del $80 \%$ de los cánceres de endometrio son típicamente adenocarcinomas. El amplio espectro de agresividad en los tipos histológicos del cáncer endometrial, según la OMS incluye: adenocarcinoma endometroide, carcinoma mucinoso, carcinoma seroso papilar $(<10 \%)$, carcinoma de células claras $(4 \%)$, carcinoma epidermoide, carcinoma de células mixtas $(10 \%)$, y carcinoma indiferenciado ${ }^{18,19}$.

Ental sentido, el objetivo del presente estudio es determinarla prevalencia del tipo histológico del endometrio en pacientes que presentan sangrado uterino postmenopáusico que han sido atendidas en el Servicio de Ginecología del Instituto Nacional Materno Perinatal de Lima.

\section{MATERIALES Y METODOS}

Estudio observacional, descriptivo, retrospectivo y transversal, que incluyeron 40 historias clínicas de pacientes atendidas en el Servicio de Ginecología del Instituto Nacional Materno Perinatal, que presentaron diagnóstico de sangrado postmenopaúsico, que fueron sometidas a intervención quirúrgica de biopsia de endometrio mediante Aspiración Manual Endouterina (AMEU) o legrado uterino instrumental (LUI), cuyo resultado histopatológico fue examinado en el Servicio de Anatomía Patológica. Los criterios de inclusión corresponden a pacientes cuyas edades son de 50 a más años, sometidas a estudio endometrial, durante el periodo comprendido entre junio 2013 a junio 2015. Se excluyeron los casos que recibieron terapia hormonal y las muestras de endometrio insuficientes que no permitieron el diagnóstico histológico.

\section{RESULTADOS}

Se aprecia en la tabla 1 que se han estudiado 40 casos de pacientes con sangrado postmenopáusico de los cuales la mayoría (77\%)corresponden a las edades comprendidas entre 50 a 59 años de edad, seguida de 65 a más años de edad (15\%).

Tabla 1. Prevalencia de sangrado uterino en la postmenopausia según grupo etario. Instituto Nacional Materno Perinatal. Periodo Junio 2013- Junio 2015

\begin{tabular}{ccc}
\hline Edad (años) & Total & Porcentaje \\
\hline $50-54$ & 22 & 55 \\
$55-59$ & 9 & 22 \\
$60-64$ & 3 & 8 \\
65 a + & 6 & 15 \\
Total & 40 & $100 \%$ \\
\hline
\end{tabular}

Los resultados de histopatología del endometrio en 40 casos estudiados en pacientes con sangrado postmenopáusico muestran que la mayoría de ellas $45 \%$ ( 18 casos) tienen endometrio normal, seguida de $15 \%$ ( 6 casos) cáncer endometrial, 13\% (5 casos) endometrio atrófico, $7 \%$ ( 3 casos) pólipo endometrial y $5 \%$ ( 2 casos) hiperplasia endometrial. Asimismo, cabe señalar que el $15 \%$ de muestras obtenidas fueron muestras insuficientes que no permitieron arribar al diagnostico histológico.

La atrofia endometrial se halló en todos los grupos etarios estudiados, mientras la hiperplasia endometrial y el pólipo endometrial se presentan con más frecuencia entre 50 a 59 años de edad.

Es importante resaltar que el tipo histológico de cáncer endometrial encontrado corresponden: carcinoma endometrial ( 2 casos), carcinoma epidermoide ( 2 casos), adenocarcinoma (1 caso) y un caso de carcinoma seroso

Tabla 2. Histopatología del endometrio en pacientes con sangrado uterino en la postmenopausia. Instituto Nacional Materno Perinatal. Periodo Junio 2013-Junio 2015.

\begin{tabular}{|c|c|c|c|c|c|c|c|}
\hline $\begin{array}{l}\text { Edad } \\
\text { (años) }\end{array}$ & $\begin{array}{c}\text { Endometrio } \\
\text { Normal } \\
\text { Sin Atipia }\end{array}$ & $\begin{array}{c}\text { Endometrio } \\
\text { Atrófico }\end{array}$ & $\begin{array}{l}\text { Hiperplasia } \\
\text { Endometrial }\end{array}$ & $\begin{array}{c}\text { Pólipo } \\
\text { Endometrial }\end{array}$ & $\begin{array}{c}\text { Cáncer } \\
\text { Endometrial }\end{array}$ & $\begin{array}{c}\text { Muestra } \\
\text { Insuficiente }\end{array}$ & Total \\
\hline $50-54$ & 11 & 2 & 1 & 2 & 3 & 3 & 22 \\
\hline $55-59$ & 6 & 1 & 1 & 1 & 0 & 0 & 9 \\
\hline $60-64$ & 1 & 1 & 0 & 0 & 0 & 1 & 3 \\
\hline $65 a+$ & 0 & 1 & 0 & 0 & 3 & 2 & 6 \\
\hline Total & 18 & 5 & 2 & 3 & 6 & 6 & 40 \\
\hline$\%$ & 45 & 13 & 5 & 7 & 15 & 15 & 100 \\
\hline
\end{tabular}


Tabla 3. Tipo histológico de cáncer endometrial en pacientes con sangrado uterino en la postmenopausiaInstituto Nacional Materno Perinatal- Periodo Junio 2013-Junio 2015.

\begin{tabular}{cl}
\hline $\begin{array}{c}\text { Edad } \\
\text { (años) }\end{array}$ & \multicolumn{1}{c}{ Tipo histológico de Cáncer Endometrial } \\
\hline 51 & Carcinoma endometrial \\
51 & Carcinoma epidermoide moderadamente diferenciado. \\
53 & Carcinoma epidermoide infiltrante moderadamente \\
66 & Carerenciado. \\
66 & Adenocarcinoma endometrial pobremente diferenciado \\
69 & Carcinoma seroso papilar \\
\hline
\end{tabular}

papilar. La prevalencia del cáncer endometrial encontrada fue en la postmenopausia temprana ( 50-54 años de edad) y después de los 65 años de edad.

\section{DISCUSIÓN}

El sangrado vaginal proveniente del útero en mujeres postmenopaúsicas deben poner en alerta al Médico Gíneco-Obstetra ya que puede ser la primera manifestación clínica de una lesión neoplásica cuyo origen puede provenir de endometrio, miometrio, ovarios, cérvix y muy rara vez de trompa de Falopio.

Según la literatura se estima que alrededor de una cuarta parte de las mujeres padecerán un sangrado uterino anormal pre o postmenopáusico en algún momento de su vida ${ }^{20}$. Asimismo, el riesgo de cáncer endometrial por 100.000 mujeres aumenta con la edad: 2,8 entre los 30 y 34 años; 6,1 entre los 35 y 39 años; y 36,5 entre los 40 y 49 años ${ }^{18}$.

Últimas publicaciones mencionan que aproximadamente $10 \%$ de las mujeres que experimentan sangrado postmenopáusico presentarán cáncer endometrial, sin embargo a pesar de ser un síntoma alarmante, la mayoría de las causas suelen ser benignas o atrofia endometrial $7,11,12$.

Los hallazgos en nuestro estudio muestran que la mayoría de casos $(45 \%)$ corresponden a endometrio normal, seguida de $15 \%$ cáncer endometrial, $13 \%$ endometrio atrófico, $7 \%$ pólipo endometrial y $5 \%$ hiperplasia endometrial; que difieren con otros estudios, como de Prendergast EN et al $^{7}$, que demuestran en una serie de 1.138 mujeres de 41 a 91 años de edad, con sangrado posmenopáusico reportan los siguientes tipos y frecuencia de histopatología7: atrofia $(59 \%)$, los pólipos $(12 \%)$, cáncer de endometrio (10\%), hiperplasia endometrial $(9,8 \%)$, efecto hormonal $(7 \%)$, cáncer cervical $(<1 \%)$. Asimismo, otros autores ${ }^{15,21}$ informan que la hemorragia uterina posmenopáusica por lo general es causada por atrofia del endometrio en el $60-80 \%$, pólipos endometriales entre $2-12 \%$, hiperplasia endometrial entre $5-10 \%$ y tratamiento estrogénico de sustitución entre 15-
$25 \%$; sin embargo, en este grupo son más frecuentes las neoplasias malignas, especialmente el carcinoma endometrial.

La atrofia de la vagina y endometrio es causada por hipoestrogenismo. En el útero, las superficies, de endometrio atrófico colapsadas contienen poco o nada de fluido para evitar la fricción intracavitaria ${ }^{22}$. Esto se traduce en microerosiones del epitelio superficial y una reacción inflamatoria crónica posterior (endometritis crónica), que es propenso a sangrado ligero o manchado (spotting).

Los pólipos endometriales son una de las causas más comunes de sangrado genital anormal tanto en las mujeres premenopáusicas y posmenopáusicas ${ }^{23-25}$. Son crecimientos hiperplásicas de glándulas endometriales y estroma que forman una proyección de la superficie del endometrio . Ellos también pueden ser asintomáticas. La gran mayoría de los pólipos endometriales son benignos, pero malignidad se produce en algunas mujeres ${ }^{24}$.

La hiperplasia endometrial se caracteriza por una proliferación de glándulas endometriales de tamaño y forma irregular. En comparación con endometrio proliferativo, hay un aumento en la glándula endometrial a la proporción de estroma. Hiperplasia endometrial casi siempre resulta de la estimulación crónica de estrógenos sin oposición por los efectos de contrapeso de progesterona ${ }^{26}$.

Según la OMS la hiperplasia endometrial se clasifica en 4 categorías: hiperplasia simple sin atipia, hiperplasia compleja sin atipia, hiperplasia atípica simple y hiperplasia atípica compleja ${ }^{27}$.

Hiperplasia endometrial generalmente se presenta con sangrado uterino anormal y es más común en las mujeres posmenopáusicas. Dado que las mujeres posmenopáusicas son deficientes en estrógenos, la hiperplasia endometrial en este momento es anormal y requiere una explicación. La producción de estrógeno endógeno de los tumores ováricos o suprarrenales o la terapia de estrógenos exógenos son posibles causas. Las mujeres obesas también tienen altos niveles de estrógenos endógenos debido a la conversión de androstendiona en estrona y la aromatización de los andrógenos a estradiol, ambos de los cuales se producen en el tejido adiposo periférico.

Según datos disponibles de un estudio ${ }^{28}$ la incidencia global de la hiperplasia endometrial fue de 133 por 100.000 años-mujer. El diagnóstico se realiza con mayor frecuencia en la mujer de 50 a 54 años de edad y rara vez se encontró en las mujeres menores de 30 años de edad, la incidencia de hiperplasia simple y compleja sin atipia fueron más altas en las mujeres de edad 50 a 54 años (142 y 213 por 100.000 mujer- año, respectivamente), mientras que la tasa de hiperplasia atípica fue más alto 
en las mujeres de 60 a 64 años de edad (56 por 100.000 años-mujer).

Con relación al cáncer endometrial en nuestra casuística se hallaron una prevalencia del $15 \%$ ( 6 casos) en relación al total de casos estudiados; de los cuales corresponden a los siguientes tipos histológico: carcinoma endometrial ( 2 casos), carcinoma epidermoide ( 2 casos), 1 caso de adenocarcinoma y un carcinoma seroso papilar; los cuales difieren a lo descrito por otros autores ${ }^{15,18,19}$ quienes reportan que más del $80 \%$ de los cánceres de endometrio son típicamente adenocarcinomas y el amplio espectro de agresividad en los tipos histológicos del cáncer endometrial, según la Organización Mundial de la Salud incluyen: adenocarcinoma endometroide, carcinoma mucinoso, carcinoma seroso papilar $(<10 \%)$, carcinoma de células claras $(4 \%)$, carcinoma epidermoide, carcinoma de células mixtas (10\%), y carcinoma indiferenciado ${ }^{15,18,19}$.

Según recientes publicaciones ${ }^{24,}{ }^{29-33}$ aproximadamente un 5 a 10 por ciento de las mujeres con sangrado vaginal después de la menopausia tienen cáncer de endometrio. Cuya incidencia se incrementa con el aumento de la edad postmenopáusica. El cáncer genital más común en mujeres más de 45 años de edad es el adenocarcinoma de endometrio. Otros autores como, Munro MG ${ }^{9}$ aplicando un modelo utilizando solo la historia clínica para predecir el riesgo de cáncer de endometrio en las mujeres post o perimenopáusicas con sangrado uterino anormal demostró que las mujeres nulíparas mayores de 70 años con diabetes mellitus presentaban 87 por ciento de riesgo de hiperplasia compleja o cáncer de endometrio en comparación al 3 por ciento en mujeres sin estas características ${ }^{9}$.

No olvidar en las mujeres con hemorragia posmenopáusica y sin terapia de reemplazo hormonal, la evaluación endometrial mediante la ecografía transvaginal un grosor endometrial de menos de o igual a 4 o $5 \mathrm{~mm}$ se asocia con un bajo riesgo de enfermedad endometrial ${ }^{34,35}$.

Estas recomendaciones se apoyan en meta-análisis de 35 estudios prospectivos ${ }^{36}$ que incluyó datos de casi 6.000 mujeres con sangrado posmenopáusico. La sensibilidad y especificidad de ecografía transvaginal para la detección de cáncer de endometrio en un umbral de $4 \mathrm{~mm}$ de grosor endometrial fueron del 96 y 53 por ciento respectivamente, y en un umbral de $5 \mathrm{~mm}$ de espesor endometrial la sensibilidad y especificidad fueron del 96 y 61 por ciento respectivamente. Además en dicha publicación ${ }^{36}$ calculan que la probabilidad de cáncer de endometrio (basado en el promedio de riesgo asociado con sangrado posmenopáusico) fue de 1 por ciento después de una ecografía transvaginal normal ( $<5 \mathrm{~mm}$ de grosor endometrial). Por eso, para evaluar el sangrado posmenopáusico la meta más importante en el uso de ecografía transvaginal es la maximización de la sensibilidad, ya que la prioridad es detectar todos los cánceres.
Finalmente, señalar que la biopsia endometrial debe ser prueba inicial preferida para las mujeres con sangrado uterino anormal debido a su alta sensibilidad, bajo índice de complicaciones, y bajo costo.

\section{REFERENCIAS BIBLIOGRÁFICAS}

1. Breijer M. Diagnostic Strategies for Postmenopausal Bleeding. Obstetrics and Gynecology International 2010: 1155.

2. Ayala F. Climaterio y Menopausia: Perspectiva de manejo en la salud femenina. Editorial CONCYTEC. Primera edición. Perú 2006 pp 145-151.

3. Astrup K, Olivarius Nde F. Frequency of spontaneously occurring postmenopausal bleeding in the general population. Acta Obstet Gynecol Scand 2004; 83:203.

4. Rossouw JE, Anderson GL, Prentice RL, et al. Risks and benefits of estrogen plus progestin in healthy postmenopausal women: principal results From the Women's Health Initiative randomized controlled trial. JAMA 2002; 288:321.

5. Smith-Bindman R, Weiss E, Feldstein V. How thick is too thick? When endometrial thickness should prompt biopsy in postmenopausal women without vaginal bleeding. Ultrasound Obstet Gynecol 2004; 24:558

6. IMirkin S, Archer DF, Taylor HS, et al. Differential effects of menopausal therapies on the endometrium. Menopause 2014; 21:899.

7. Prendergast EN, Misch E, Chou YA, et al. Insufficient endometrial biopsy results in women with abnormal uterine bleeding. Obstet Gynecol 2014; 123 Suppl 1:180S.

8. David $F$ et al. Endometrial bleeding in postmenopausal women:with and without hormone therapy. The Journal of The North American Menopause Society 2011: 416-420.

9. Munro MG, Southern California Permanente Medical Group's Abnormal Uterine Bleeding Working Group. Investigation of women with postmenopausal uterine bleeding: clinical practice recommendations. Perm J 2014; 18:55.

10. Prendergast EN, Misch E, Chou YA, et al. Insufficient endometrial biopsy results in women with abnormal uterine bleeding. Obstet Gynecol 2014; 123 Suppl 1:180S.

11. Smith PP, O'Connor S, Gupta J, Clark TJ. Recurrent postmenopausal bleeding: a prospective cohort study. J Minim Invasive Gynecol 2014; 21:799.

12. Munot S. Modern Management of Postmenopausal bleeding. Trends in Urology Gynaecology \& Sexual Health 2008: 20-24.

13. APGO educational series on women's health issues. Clinical management of abnormal uterine bleeding. Association of Professors of Gynecology and Obstetrics, 2006.

14. Lieng M, Istre O, Qvigstad E. Treatment of endometrial polyps: a systematic review. Acta Obstet Gynecol Scand 2010; 89:992.

15. Shorge J, Schaffer J. Hemorragia uterina anormal. Ginecología de Williams. 1era ed. México, D.F. McGraw)Hill Interamericana Editores. 2009. Págs. 174-190, 896, 897, 950-953

16. Socías $T$, Vargas $R$, et al. Correlación de la Histeroscopía y Biopsia dirigida en el estudio del Engrosamiento Endometrial Patológico por Ultrasonido. Revista Chilena de Obstetricia y Ginecología. Santiago de Chile, 2007. 72 (2): 99-104.

17. Bankowsky B, Hearne A, Lambrou N, Fox H, Wallach E, Johns Hopkins. Manual de Ginecología y Obstetricia. 2da Edición. Madrid, España. Editorial Marbán, 2005. Págs., 405411. 
18. Ely J, Kennedy C, Clark E, Bowdler N. Abnormal Uterine Bleeding: A Management Algorithm. J Am Board Family Med 2006; 19(6):590- 602. Disponible en: http://www.jabfm.org/ cgi/reprint/19/6/590 Consultado Febrero 9, 2011

19. American Cancer Society. Cáncer de endometrio (uterino). Disponible en: http://www.cancer.org/Espanol/cancer/ Cancerdeendometrio/Guiadetallada/cancer-de-endometriouterino-what-is-key-statistics\#top. 2011. Consultado Junio 7, 2011

20. González A, Rodríguez A. Polipectomía Histeroscópica. Servicio de Obstetricia y Ginecología. Hospital Universitario Virgen de las Nieves Granada. Pág. 2. Andalucía, España, 2007. Disponible en: www.hvn.es/servicios.../ginecologia... obstetricia/.../cr07.polipectomia_histeroscopica.pdf Consultado Enero 15, 2011

21. Espindola D, Kennedy K, Fischer E. Tratamiento de la hemorragia uterina anormal y patología de la hiperplasia endometrial. Clínicas Obstétricas y Ginecológicas de Norteamérica. USA, 2007. Elsevier Saunders. Págs.717 737

22. Ferenczy A. Pathophysiology of endometrial bleeding. Maturitas 2003; 45:1.

23. Lieng $M$, Istre $O$, Qvigstad E. Treatment of endometrial polyps: a systematic review. Acta Obstet Gynecol Scand 2010; 89:992.

24. Lee SC, Kaunitz AM, Sanchez-Ramos L, Rhatigan RM. The oncogenic potential of endometrial polyps: a systematic review and meta-analysis. Obstet Gynecol 2010; 116:1197.

25. Mutter GL, Nucci, MR, Robboy SJ. Endometritis, metaplasias, polyps, and miscellaneous changes. In: Robboy's Pathology of the Female Reproductie Tract, 2nd ed., Robboy SJ, Mutter GL, Prat J, et al. (Eds), Churchill Livingston Elsevier, Oxford 2009. p.343.

26. Hedrick Ellenson L, Ronnett BM, Kurman RJ. Precursor Lesions of Endometrial Carcinoma. In: Blaustein's Pathology of the Female Genital Tract, 6th ed, Kurman RJ, Hedrick
Ellenson L, Ronnett, BM. (Eds), Springer, New York 2010 p.360-361.

27. Scully RE, Bonfiglio TA, Kurman, et al. Uterine corpus. In: Histological Typing of Female Genital Tract Tumours, 2nd ed. Springer-Verlag, New York 1994. p.13.

28. Reed SD, Newton KM, Clinton WL, et al. Incidence of endometrial hyperplasia. Am J Obstet Gynecol 2009; 200:678.e1.

29. Burbos N, Musonda P, Giarenis I, et al. Age-related differential diagnosis of vaginal bleeding in postmenopausal women a series of 3047 symptomatic postmenopausal women. Menopause Int 2010; 16:5

30. Bani-Irshaid I, Al-Sumadi A. Histological findings in women with postmenopausal bleeding: Jordanian figures. East Mediterr Health J 2011; 17:582.

31. Ronghe R, Gaudoin M. Women with recurrent postmenopausal bleeding should be re-investigated but are not more likely to have endometrial cancer. Menopause Int 2010; 16:9.

32. Van Hanegem N, Breijer MC, Khan KS, et al. Diagnostic evaluation of the endometrium in postmenopausal bleeding an evidence-based approach. Maturitas 2011; 68:155.

33. Cote ML, Ruterbusch JJ, Ahmed Q, et al. Endometrial cancer in morbidly obese women: do racial disparities affect surgical or survival outcomes? Gynecol Oncol 2014; $133: 38$.

34. Goldstein SR, Nachtigall M, Snyder JR, Nachtigall L. Endometrial assessment by vaginal ultrasonography before endometrial sampling in patients with postmenopausal bleeding. Am J Obstet Gynecol 1990; 163:119.

35. Karlsson B, Granberg S, Wikland M, et al. Transvaginal ultrasonography of the endometrium in women with postmenopausal bleeding--a Nordic multicenter study. Am J Obstet Gynecol 1995; 172:1488.

36. Smith-Bindman R, Kerlikowske K, Feldstein VA, et al Endovaginal ultrasound to exclude endometrial cancer and other endometrial abnormalities. JAMA 1998; 280:1510. 